\title{
Employment changes during the COVID-19-pandemic and mental health: Evidence from a longitudinal study
}

\author{
Bjørn-Atle Reme ${ }^{1, \dagger}$, Jonathan Wörn ${ }^{1, \dagger}$ and Vegard Skirbekk ${ }^{1,2}$ \\ Preprint \\ available on https://osf.io/4nu7c \\ doi: 10.31219/osf.io/4nu7c
}

December 7, 2021

This manuscript is currently under revision. Please do not cite. 